\title{
Ultrasonographic findings in Fitz-Hugh-Curtis syndrome: a thickened or three-layer hepatic capsule
}

\author{
You Ho Moon 1 , Jung ho Kim², Won joon Jeong ${ }^{3}$, Sin-Youl Park ${ }^{2}$ \\ ${ }^{I}$ Department of Emergency Medicine, Daegu Fatima Hospital, Daegu; ${ }^{2}$ Department of Emergency Medicine, Yeungnam University \\ College of Medicine, Daegu; ${ }^{3}$ Department of Emergency Medicine, Chungnam National University Hospital, Daejeon, Korea
}

Fitz-Hugh-Curtis syndrome (FHCS) is characterized by inflammation of the perihepatic capsules associated with the pelvic inflammatory disease (PID). FHCS is not a serious disease, but if not treated properly, it can result in increased medical costs, prolonged treatment, and dissatisfaction with treatment. However, early recognition of FHCS in the emergency department can be difficult because its symptoms or physical findings may mimic many other diseases. Although contrast-enhanced computed tomography (CECT) is the useful imaging modality for recognition of FHCS, it is available only when a high suspicion is established. We performed point-of-care ultrasonography in an 18-year-old woman who had a sharp right upper quadrant (RUQ) abdominal pain without PID symptoms and found a thickened or three-layer hepatic capsule. These findings coincided with areas showing increased hepatic capsular enhancement in the arterial phase of CECT. These results show that if the thickened or three-layer hepatic capsule without evidence of a common cause of RUQ pain is observed on ultrasonography in women of childbearing age with RUQ abdominal pain, the physician can consider the possibility of FHCS.

Keywords: Pelvic inflammatory disease; Ultrasonography; Abdominal pain

\section{INTRODUCTION}

Patients with abdominal pain localized in the right upper quadrant (RUQ) of the abdomen are common in the emergency department (ED). Various conditions can cause RUQ pain. Fitz-Hugh-Curtis syndrome (FHCS) is one of the rare causes of RUQ abdominal pain.

FHCS is characterized by inflammation of the hepatic capsules in pelvic inflammatory disease (PID) [1]. The pathoge-

Received: April 24, 2018, Revised: April 29, 2018

Accepted: April 30, 2018

Corresponding Author: Sin-Youl Park, Department of Emergency Medicine, Yeungnam University College of Medicine, 170, Hyeonchung-ro, Nam-gu, Daegu 42415, Korea

Tel: +82-53-620-3626, Fax: +82-53-623-8030

E-mail: dryuri@naver.com nesis of FHCS is poorly understood, but its incidence was reported to be $4-27 \%$ in patients with PID [2]. The typical symptoms include a sudden sharp pain in the RUQ of the abdomen, combined with fever, lower abdominal pain, and vaginal discharge, but may present with only RUQ abdominal pain $[1,2]$. The diagnosis is made when violin-like adhesions are detected or causative organisms (Chlamydia trachomatis or Neisseria gonorrhoeae) are identified in the hepatic capsular lesion specimens. However, generally, the diagnosis is made clinically on the basis of symptoms or physical examinations, and the exclusion of other causes of RUQ abdominal pain [2]. However, early recognition of FHCS can be difficult because its symptoms or physical findings may mimic many other diseases. We will discuss the early recognition of FHCS in the ED through a case report of a young woman with RUQ pain.

Copyright (C) 2018 Yeungnam University College of Medicine

This is an Open Access article distributed under the terms of the Creative Commons Attribution Non-Commercial License (http://creativecommons.org/licenses/by-nc/4.0/) which permits unrestricted non-commercial use, distribution, and reproduction in any medium, provided the original work is properly cited. 


\section{CASE}

An 18-year-old woman undergoing management of cystitis presented to the ED with a squeezing and sharp pain in the RUQ of the abdomen for 3 days. The pain was aggravated by breathing. Physical examination revealed maximal pain and tenderness in the RUQ of the abdomen. No pain and tenderness were observed in the lower abdomen. The vital signs were stable without fever. The blood test results were unremarkable, except for the C-reactive protein levels (21 $\mathrm{mg} / \mathrm{dL}$ ). Urinalysis detected 10-20 leucocytes. Point-of-care ultrasonography (POCUS) in the RUQ abdomen and contrastenhanced computed tomography (CECT) were performed sequentially (Fig. 1). On POCUS, no abnormal findings were observed in the gallbladder, hepatic parenchyma, and right pleural space. On the other hand, in the right subcostal scan, the hepatic capsule was observed to be abnormally bright and thickened (Fig. 1A). According to the scan angle and the patient's respiration, the thickened hepatic capsule was observed as three layers (hyperechoic layer-hypoechoic layerhyperechoic layer; Fig. 1B). In the arterial phase of the CECT, hepatic capsular enhancements were observed on the right side of the liver (Fig. 1C). The patient was diagnosed as having FHCS on the basis of her symptoms, physical examination, and imaging findings. She was treated in accordance with the PID treatment guidelines. After 3 days of oral antibiotic therapy, she visited the outpatient clinic without symp- toms. The treatment was completed without complications.

\section{DISCUSSION}

FHCS is not a serious disease, but if not properly diagnosed, it can result in increased medical costs, prolonged treatment, and dissatisfaction with treatment. Increased hepatic capsular enhancement in the arterial phase of CECT is useful for the recognition of FHCS in the ED [3]. However, it is available only when the index of suspicion is high.

The ultrasonographic findings of FHCS may be normal, but a report indicated that a thickened hepatic capsule and ascites could be observed on ultrasonography [4]. Given the pathophysiology of FHCS, ultrasonographic images that suggest perihepatitis is an important finding in the recognition of FHCS. Unfortunately, ultrasonographic images of patients with FHCS that show inflammation and ascites between the liver and the diaphragm or abdominal wall are rarely observed.

In the present patient with FHCS, POCUS performed on the RUQ abdomen revealed a thickened and three-layer hepatic capsule between the liver and the diaphragm and abdominal wall. A comparison with the CECT images suggested that the inner hyperechoic, middle hypoechoic, and outer hyperechoic layers of the hepatic capsule observed on ultrasonography indicated the inflammation in the liver surface, a small amount of ascites, and the inflammation in the diaphragm and abdominal wall, respectively. These abnormal ultrasono-
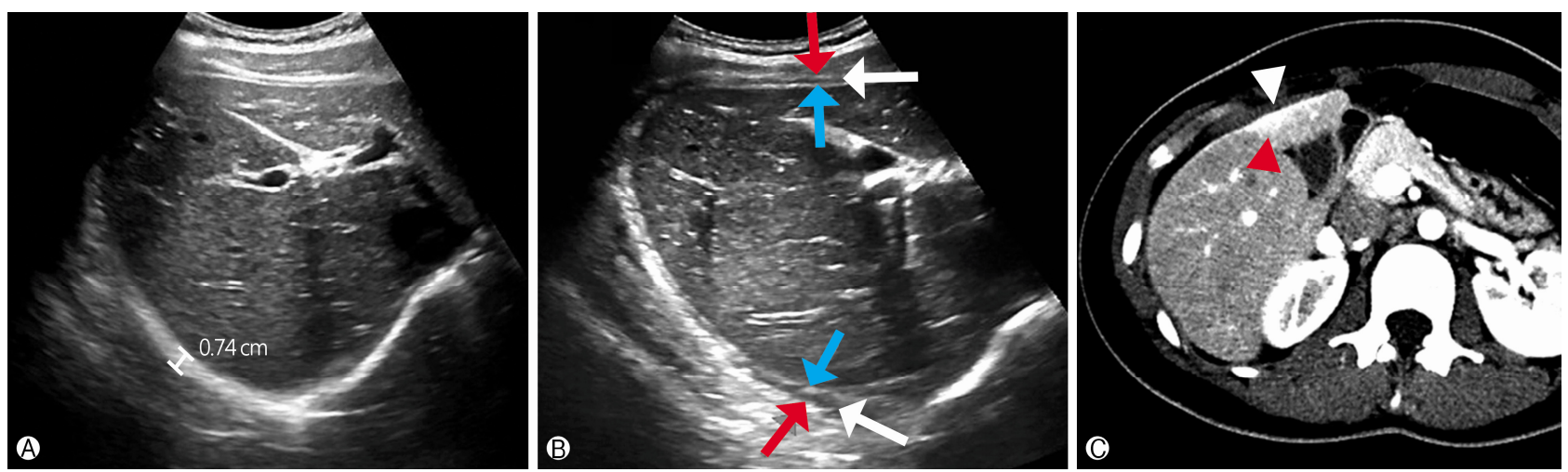

Fig. 1. POCUS and CECT findings of the patient. (A) Right subcostal POCUS scan showing abnormal thickening $(0.74 \mathrm{~cm})$ between the liver and the diaphragm. (B) According to the scan angle and respiration, the hepatic capsule is shown as three layers on the right subcostal scan. The inner hyperechoic (blue arrow), middle hypoechoic (white arrow), and outer hyperechoic layers (red arrow) of the hepatic capsule are thought to indicate the inflammation of the liver surface, a small amount of ascites, and the inflammation of the diaphragm or abdominal wall, respectively. (C) In the arterial phase of the CECT scan, hepatic capsular enhancement (red arrowhead) and a small amount of localized ascites (white arrowhead) were observed on the surface of segment IV of the liver. POCUS, point-of-care ultrasonography; CECT, contrast-enhanced computed tomography. 
graphic findings in the hepatic capsule may also be found in other diseases that cause perihepatitis, such as perforated cholecystitis, perforated hepatic abscess, tuberculous peritonitis, direct radiation exposure of the liver, and peritoneal carcinomatosis [5]. However, these diseases have distinct differences from FHCS in terms of medical history, symptoms, physical examination results, underlying disease, and combined disease.

Ultrasonography is well known to be useful in excluding the common causes of RUQ pain. The present case shows that perihepatitis in FHCS can be observed as a thickened or threelayer hepatic capsule on ultrasonography. These results show that if a thickened or three-layer hepatic capsule without evidence of a common cause of RUQ abdominal pain is shown on ultrasonography in women of childbearing age with RUQ pain, the emergency physician can consider the possibility of FHCS.

\section{CONFLICT OF INTEREST}

No potential conflict of interest relevant to this article was reported.

\section{ORCID}

You Ho Moon, https://orcid.org/0000-0003-0585-8674

Sin-Youl Park, https://orcid.org/0000-0003-4005-1956

\section{REFERENCES}

1. Fitz-Hugh T. Acute gonococcic peritonitis of the right upper quadrant in women. J Am Med Assoc 1934;102:2094-6.

2. Peter NG, Clark LR, Jaeger JR. Fitz-Hugh-Curtis syndrome: a diagnosis to consider in women with right upper quadrant pain. Cleve Clin J Med 2004;71:233-9.

3. Tsubuku M, Hayashi S, Terahara A, Furukawa T, Ohmura G. Fitz-Hugh-Curtis syndrome: linear contrast enhancement of the surface of the liver on CT. J Comput Assist Tomogr 2002; 26:456-8.

4. van Dongen PW. Diagnosis of Fitz-Hugh-Curtis syndrome by ultrasound. Eur J Obstet Gynecol Reprod Biol 1993;50:15962.

5. Kim S, Kim TU, Lee JW, Lee TH, Lee SH, Jeon TY, et al. The perihepatic space: comprehensive anatomy and CT features of pathologic conditions. Radiographics 2007;27:12943. 